\title{
VIDA O MUERTE DEL CRIOLLO LIMONENSE
}

\section{Anita Herzfeld *}

En un ensayo titulado "Las prioridades en los estudios de los idiomas criollos" (Pidgins and Creoles: Current Trends and Prospects, eds. David DeCamp e Ian F. Hancock, 1974, p. 86), Derek Bickerton recomienda que los lingüistas estudien "las variaciones que se encuentran tanto en el lenguaje del grupo como en el del individuo en muchas comunidades de habla criolla". Uno de los propósitos de esta ponencia es el de describir la variación de un idioma criollo en interacción con la lengua de prestigio en una comunidad.

El Criollo Limonense (que se habla en la costa Caribeña de Costa Rica) desciende del Criollo Jamaiquino y es muy similar a éste en muchos sentidos. Mientras que el Criollo Jamaiquino ha sido muy cuidadosamente estudiado, son pocos los trabajos sobre un criollo inglés en el contexto de un idioma de prestigio no relacionado con la base del criollo -en este caso, el castellano, idioma nacional de Costa Rica. Desearía demostrar que el castellano de Costa Rica influye en el léxico, las estructuras sintácticas y el alcance semántico del Criollo Limonense. En otras palabras, aunque el inglés se mantiene aún como una de las lenguas de prestigio (el idioma "standard" de la variedad "acrolectal") el castellano también afecta al criollo. Lo hace, especialmente, en las variedades del mesolecto y del basilecto. En consecuencia, el Criollo Limonense cuenta hoy con gran cantidad de préstamos españoles, a ilustrarse en este trabajo. ¿Podría uno aventurarse a predecir cómo va a afectar este proceso el futuro del Criollo Limonense?

\section{INTRODUCCION}

Costa Rica, una de las cinco repúblicas de Centro América, ha albergado a una minoría negra durante 400 años. Esta población, que desde el si-

* $\quad$ Profesora en la Universidad de Kansas, U.S.A. glo XIX practica la religión protestante y habla un idioma criollo de base inglesa, ha vívido siempre en las tierras bajas de la costa Atlántica (Provincia de Limón), mientras que la sociedad que se considera "verdadero" exponente de la cultura costarricense -blanca, católica, y de habla hispana- habita en las tierras altas (meseta central). Michael D. Olien (uno de los pocos antropólogos que ha estudiado esta población) reconoce tres cambios estructurales importantes en la sociedad costarricense que resultaron en alteraciones de consideración en la posición del Negro: (1)

1. la polarización del poder que tuvo lugar durante y poco después del período colonial (1570-1870),

2. el control de facto de las tierras bajas ejercido por la United Fruit Co, (1870-1948), y

3. las reformas legales y sociales instituidas por la revolución de 1948 (1948-presente).

Correspondiendo a cada uno de los periodos mencionados, Olien distingue la existencia de tres tipos de negros. Son respectivamente, los siguientes:

1. el negro africano

2. el negro de las Indias Occidentales

3. el negro costarricense.

Sostiene Olien que estos tres tipos diferentes están directamente relacionados con diversos patrones de adaptación a la cultura costarricense. El negro africano del primer período desapareció como resultado de la mezcla de razas, de modo que no hubo continuidad o secuencia evolutiva en el pasaje de la primera etapa de su presencia a la otra. Por otra parte, el negro de las Indias Occidentales (que vivió en Costa Rica a fines del siglo XIX y a comienzos del XX) ejemplifica el patrón de separatismo que se dió durante el auge de la United Fruit Co. En su mayoría este contingente estaba 
compuesto de campesinos jamaicanos que se establecieron temporariamente en Costa Rica para trabajar con la UFCo. (primero en el ferrocarril, luego en las plantaciones bananeras, pero siempre pensando en regresar a su patria). Su aculturación a Costa Rica transcurrió con suma lentitud -más bien fue demorada adrede - porque el "gobierno indiscutiblemente monopolista, totalitario e imperialista" (Bryce Laporte, 1962:6) ejercido por los inversores extranjeros sobre los trabajadores para reforzar su propio poder, fomentó la alienación de los mismos del resto del país Estimulados por "Mamita Yunai" (2) los trabajadores así coercionados, no tuvieron inconveniente en conservar el idioma inglés y su religión protestante, cumpliendo de este modo con las directivas de la UFCo. No es nada sorprendente, pues, que el inglés adquiriera entonces un lugar destacado en la vida de la región y del criollo jamaicano. Resultó ser el medio de comunicación en la vida de la familia y de la comunidad, para la mayoría de la población negra de Limón. Esto dió lugar a la existencia de un continuo de variación: el criollo limonense. (3)

El cuadro de unidad lingüística que caracteriza al negro del segundo período comienza a desbaratarse en el tercero. Nuevos grupos nacionales de prestigio y de poder surgen en Costa Rica como consecuencia de la revolución del 48. El negro costarricense abandona el sistema de plantación para compenetrarse gradualmente de las costumbres del país: adopta la nacionalidad costarricense, manda a sus hijos a las escuelas públicas de habla hispana, aprende a hablar el español, y algunos hasta se convierten al catolicismo.

Cuando la compañía bananera elimina sus operaciones en la costa Atlántica (1947), numerosos contingentes de blancos costarricenses de habla hispana se establecieron en Limón. Hoy día, los blancos son mayoría en esta zona. El inglés ha perdido influencia y el criollo limonense ha sufrido un gran cambio en sus funciones sociales de comunicación. Ahora el idioma de prestigio es el castellano, que se usa como medio de instrucción, de comunicación en la radio y en los periódicos, en el comercio, en la política, en los trámites oficiales y legales, en Ios servicios públicos, etc.

El criollo limonense, conocido como mekaytelyu/, "patois," dialecto, "flat English" o "broken English", desciende del criollo jamaicano y se le parece en muchos aspectos. Mientras que el criollo jamaicano ha sido estudiado cuidadosamente, son pocos los trabajos que muestran un criollo de base inglesa en el contexto de un idioma de prestigio no relacionado con el criollo -en este caso, el castellano. El trabajo que sigue enfoca la forma en que el español de Costa Rica ejerce influencia en el léxico, en el alcance semántico, y en las estructuras sintácticas y morfológicas del criollo limonense. Además se propone identificar problemas relacionados con la situación de las lenguas en contacto.

\section{LOS IDIOMAS CRIOLLOS Y EL CAMBIO EN EL LENGUAJE}

Parecen ser cuatro las áreas en que se concentran los esfuerzos de los lingüistas contemporáneos que enfocan los problemas del cambio en el lenguaje: 1) la expansión del concepto de lenguaje -que ahora incorpora el significado social del idioma. E1 1enguaje como fenómeno social recibió mucha atención durante la década anterior y la sociolingüística se ha establecido ya como rama seria de la disciplina total. 2) dentro de las corrientes modernas que redefinen el cambio en el lenguaje, figura la que sustenta la variabilidad inherente al idioma - que ha dejado de ser considerado monolítico. 3) las lenguas en contacto proveen ejemplos dramáticos de cambios en su estructura y en su uso. Se entiende que un sistema lingüistico no ha de cambiar meramente por el uso repetido de una forma determinada, sino que las bases sociales subyacentes, motivan y definen las características del cambio. 4) el renacimiento de un gran interés en la evolución del lenguaje.

Aunque los fenómenos relacionados con cambios en el lenguaje han constituido desde un principio un tema de interés en lingüística, las preguntas que se formulan hoy día, son el resultado de nuevos conceptos y de una diferente perspectiva, adoptada especialmente al estudiar lenguas en contacto (el tercer tema mencionado antes). Es precisamente el estudio de las lenguas criollas el que ha fomentado el enfoque socio-lingüístico del cambio en los idiomas. Como generalmente se hablan en comunidades que además usan otro idioma (el standard), la estructura y la variación que caracterizan a los criollos pueden relacionarse fácilmente con factores tales como la edad, el origen, el status, la educación, etc. de sus parlantes.

Este trabajo representa un estudio empírico del cambio en el lenguaje desde una perspectiva sociolingüistica. Se ocupa exclusivamente de los 
cambios de vocabulario en un criollo establecido: el limonense. La situación de contacto entre el criollo y el castellano trae aparejada un reemplazo y una reorganización del léxico, que produce divergencias en el significado y en la estructura morfológica y sintáctica del sistema lingüístico en cuestión.

\section{CAMBIOS RECIENTES EN EL LEXICO DEL CRIOLLO LIMONENSE}

Las variables sociales que se consideran importantes para determinar los efectos del español en el criollo limonense son las siguientes: (4) A. edad: dos generaciones intervinieron en este estudio. Aunque el criterio usado para definirlas es arbitrario, se basa en un rasgo significativo: un grupo generacional fue educado en escuelas particulares inglesas (el de la gente que nació antes de 1948); el otro grupo asistió a escuelas públicas costarricenses (personas nacidas después de 1948); B. educación: si un triángulo imaginario simbolizara la población total de la ciudad de Limón (5), la amplia base representaría la clase de nivel socioeconómico bajo, y el ápice la clase media, ya que no hay clase alta en Limón. La transición de una clase a la otra puede medirse exclusivamente en términos del nivel educativo logrado y de la afluencia económica. Los niveles educativos en Limón son únicamente dos: I. el nivel bajo de aquéllos que sólo han asistido a algunos grados de primaria, y II. el de los que asistieron a cursos más allá de la primaria; (6) C. sexo.

En la típica situación de un individuo que está expuesto a dos sistemas diferentes de lengua y cultura, los lingüístas y antropólogos distinguen un proceso sociológico de aprendizaje (el bilingüismo y la aculturación, respectivamente), y el cambio que es el resultado de ese proceso (la interferencia lingüística y los préstamos culturales, también respectivamente). Uriel Weinreich define "la interferencia lingüística" como "esos ejemplos de desviación de las normas de cualquiera de dos idiomas en el habla de bilingües, como resultado de su familiaridad con más de un idioma, i.e. como resultado de lenguas en contacto" (1953:1). Y como anota Bloomfield, "en todos los casos, ... es la lengua inferior la que incurre predominantemente en préstamos de la superior ..." (1933:464). La lengua superior, actúa aquí (en la forma en que Weinreich definiera el concepto de "prestigio" en situaciones multilinguales), "como una fuerza unidireccional que contribuye al avance social de una persona." (1974:79).

\section{DIVERGENCIAS EN EL SIGNIFICADO DEL LE- XICO:}

A continuación anotamos algunos ejemplos que ilustran el impacto del español (como lengua de prestigio) en el criollo limonense, a través de interferencias morfológicas de dos tipos (según una clasificación basada en Weinreich, Haugen, Diebold, y Edwards).

\section{INTERFERENCIAS MORFOLOGICAS}

I. Importaciones
A. Préstamos
1. Integración
2. Interferencia

B. Préstamos ajustados

1. Formación de nombres

2. Participio presente

II. Sustituciones
A. Préstamos-cambios
B. Sustituciones idiomáticas

\section{IMPORTACIONES Y SUSTITUCIONES}

\section{Importaciones morfológicas}
A. Préstamos

1. Integración: Los vocablos castellanos funcionan como si fueran ingleses. Ejemplos: (7)

$$
\begin{aligned}
& \text { 6.1.7 } \mathrm{O} \_- \text {a shur yu av plenti tu tel mi ... } \\
& \text { I--layk wat? } \\
& \text { O_-layk wat yu duw/we yu liv/debarrio we }
\end{aligned}
$$


2. Interferencia: El uso de vocablos españoles cuando los ingleses no vienen a la mente inmediatamente:

\subsubsection{I $\mathrm{I}-$-seben yer egow now/ay down bay now regalo fa miy fada ...} (prezen)

\section{B. Préstamos ajustados}

1. Formación de nombres: manifesteyshon, asigneyshon

2. Participio presente: confesarin, rechazarin ... (Verbo en español + -ing)

\section{Sustituciones morfológicas}

A. Préstamos-cambios: El cambio de significado de vocablos españoles que adoptan el significado inglés (falsos cognados):

6.16B $\mathrm{O}--$ wa yu gowin stodi wen yu kom owt kolij?

$($ college $=$ colegio $)$

B. Sustituciones idiomáticas: Son traducciones de expresiones idiomáticas españolas al inglés:

\subsection{3 de milk kot op} se cortó la leche

\section{DIVERGENCIAS EN LA FRECUENCIA DE IM- PORTACIONES MORFOLOGICAS}

La observación de los Cuadros $\mathrm{N}^{\circ} 1$ y $\mathrm{N}^{\circ} 2$, revela claramente que el cambio del lenguaje se produce en determinadas categorías de parlantes más frecuentemente que en otras. (8) Las personas de más edad (nacidas antes de 1948) son más conservadoras en su lenguaje en todos los niveles. Retienen en su vocabulario más términos en inglés o en criollo limonense. Los jóvenes, como era de suponer, son los principales innovadores: ellos introducen la mayor parte de los vocablos en castellano. Intimamente ligado a la edad, figura el nivel de educación adquirido, sobre todo porque 'escuela primaria' para el grupo de edad B significa la asistencia a escuelitas inglesas que existieron en Limón hasta 1953 (9). Por otra parte, la educación que han recibido los jóvenes que pertenecen a la ge- neración $\mathrm{A}$ ha tenido lugar en escuelas públicas costarricenses que imparten sus clases en español. Lógicamente, la gente mayor usa vocablos en español con menos frecuencia y los jóvenes, en especial los que han recibido más años de educación, los usan más.

Desde el punto de vista de la función que desempeñan los vocablos, se advierte que los sustantivos son los préstamos más frecuentes, tanto para las mujeres como para los hombres. Siguen luego las expresiones, los verbos, y finalmente los modificadores (adjetivos y adverbios), también en este orden para ambos sexos. Podría justificarse el frecuente uso de expresiones por tratarse de una categoría amplia que incluye cambios de idioma por múltiples razones (interlocutor, tema, inseguridad lingüística, contraste, énfasis, etc.). En cuanto a los verbos es interesante observar que en general los verbos asumen la forma del infinitivo o de la tercera persona del singular. Quizás en este caso exista una compleja superposición de reglas: el criollo no exige el uso de la $-s$ en tercera persona del singular (como sí lo haría el inglés standard: comes, writes, etc.). La tercera persona en español no necesita de un afijo tampoco, y es ésa la forma que adopta el préstamo.

En cuanto a los dos sexos, es natural que los hombres usen más vocablos en español que las mujeres: hasta recientemente pocas eran las mujeres a quienes se les permitía asistir a las escuelas, de modo que fueron muchos más los hombres que aprovecharon las clases (en ambos niveles de educación).

\section{CONCLUSIONES ACERCA DEL FUTURO DEL CRIOLLO LIMONENSE}

Resumiendo: el proceso de innovación (de importaciones morfológicas como resultado del proceso que se da entre lenguas en contacto) ha recibido relativamente poca atención de parte de los lingüistas hasta hace poco tiempo. Una de las fuentes de cambio es el préstamo de vocablos que resulta de los procesos mundiales de urbanización e integración de las sociedades locales, particularmente dictados por los sistemas de comunicación en vigencia.

Concentrándonos en el estudio de los procesos de cambio, se puede percibir cómo cambian los requisitos de comunicación y cómo las convenciones que gobiernan la feliz transmisión del mensaje con- 
ducen a innovaciones lingüísticas. El cambio puede ser estudiado entonces, observando el uso del idioma en el contexto del desarrollo social y lingüístico.

Algunas de las implicaciones teóricas de los cambios registrados en el material recogido parecen señalar que cualitativamente estos cambios no son diferentes de los que ocurren en idiomas no -criollos (por lo menos en lo que respecta a las categorías más susceptibles al traspaso). Sin embargo, difieren de los que ocurren en otros criollos, como el jamaicano, por ejemplo. En éste el acrolecto tiende hacia el inglés standard, como idioma de prestigio, y por lo tanto, busca activamente la adquisición de sus vocablos. También puede afir- marse, que no todo el vocabulario está sufriendo una masiva relexificación instantánea. (10) La observación de la categoría "sustantivo," por ejemplo, muestra que son aquellas palabras -ya sea técnicas o del medio ambiente costarricense hispanoque dominan la vida limonense, las que más fácilmente ganan entrada (barrio, redoblante, bodego, sic) en el criollo limonense. La categoría verbo incorpora términos que pertenecen a una esfera de acción "educada" (perfeccionar, expulsar, disociar).

Es probable que el extremo del acrolecto (que en otro contexto sociolingüístico se mantendría más activamente renovado por las influencias de prestigio) desaparezca aquí primero, ya que no se

\section{Cuadro $\mathrm{N}^{\circ} 1$}

\section{FRECUENCIA DE IMPORTACIONES MORFOLOGICAS ESPAÑOLAS EN EL CRIOLLO DE HOMBRES LIMONENSES}

\begin{tabular}{|c|c|c|c|c|c|c|c|c|}
\hline Lecto & Informante & Edad* & Educ** & Sustantivo & Expresión & Verbo & Modific & Sust + Mod \\
\hline \multirow[t]{7}{*}{ asilecto } & $\# 68$ & A & II & 22 & 3 & 3 & - & - \\
\hline & \# 64 & A & I & 8 & - & 1 & 1 & 3 \\
\hline & \# 191 & B & II & 14 & 2 & 1 & - & - \\
\hline & \# 66 & B & I & 5 & 4 & 2 & - & 1 \\
\hline & \multirow[t]{3}{*}{ Totales } & A & & 30 & 3 & 4 & 1 & 3 \\
\hline & & B & & 19 & 6 & 3 & - & 1 \\
\hline & & & & 49 & 9 & 7 & 1 & 4 \\
\hline \multirow[t]{7}{*}{ esolecto } & \# 141 & A & II & 6 & 5 & 1 & 2 & - \\
\hline & $\# 8$ & A & II & 14 & 2 & - & - & - \\
\hline & \# 191 & B & I & 4 & - & - & - & - \\
\hline & \# 66 & B & I & 6 & - & - & - & - \\
\hline & Totales & $\mathrm{A}$ & & 20 & 7 & 1 & 2 & - \\
\hline & & B & & 10 & - & - & - & - \\
\hline & & & & 30 & 7 & 1 & 2 & - \\
\hline \multirow[t]{3}{*}{ crolecto } & \# 204 & B & I & 1 & - & - & - & - \\
\hline & $\# 152$ & B & II & 2 & - & - & - & - \\
\hline & Totales & B & & 3 & - & - & - & - \\
\hline \multicolumn{2}{|c|}{ GRAN TOTAL } & & & 82 & 16 & 8 & 3 & 4 \\
\hline
\end{tabular}




\section{Cuadro $\mathrm{N}^{\circ} 2$}

\section{FRECUENCIA DE IMPORTACIONES MORFOLOGICAS ESPAÑOLAS EN EL CRIOLLO DE MUJERES LIMONENSES}

\begin{tabular}{|c|c|c|c|c|c|c|c|c|}
\hline Lecto & Informante & Edad* & Educ** & Sustantivo & Expresión & Verbo & Modific & Sust + Mod \\
\hline \multirow[t]{6}{*}{ asilecto } & \# 127 & $\mathbf{A}$ & II & 9 & 3 & 3 & 1 & \\
\hline & $\# 113$ & A & II & 20 & 6 & 10 & 5 & \\
\hline & \# 115 & B & I & 2 & & & & \\
\hline & \multirow[t]{3}{*}{ Totales } & $\mathbf{A}$ & & 29 & 9 & 13 & 6 & \\
\hline & & B & $\Leftrightarrow$ & 4 & & 1 & & \\
\hline & & & & 33 & 9 & 14 & 6 & \\
\hline
\end{tabular}

$\begin{array}{lll}\text { esolecto } & \text { \# 252 } & \text { A } \\ & \text { \# 268 } & \text { A } \\ \text { \# 165 } & \text { B } \\ & \text { \# 72 } & \text { B } \\ & \text { Totales } & \text { A } \\ & & \text { B }\end{array}$

\begin{tabular}{ccccc} 
II & 4 & & & \\
II & 5 & 3 & 3 & 1 \\
I & & & 1 & \\
I & 4 & 3 & 1 & 1 \\
\hline & 9 & 3 & 3 & 1 \\
& 4 & 3 & 2 & 1 \\
\hline & 13 & 6 & 5 & 2
\end{tabular}

crolecto \# 183 B

\#232 B

Totales B

\section{GRAN TOTAL}

A
A
B
B
A
B

II

II

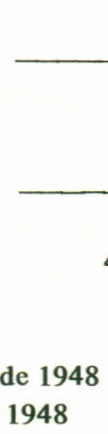

2

2

6

6

6

1

1
Edad*: $\quad$ A: nacida después de 1948

B: nacida antes de 1948

\author{
Educación**: I: menos que primaria \\ II: más que primaria
}

nutre aquí del medio ambiente (los cuadros indican escasas importaciones morfológicas hispanas en este nivel). Mientras tanto el basilecto podría sobrevivir fácilmente con una fuerte influencia española (11). El sentido común indica que el léxico "prestado" va a continuar creciendo con el tiempo, y las palabras que seguirán entrando en el idioma servirán más especialmente para precisar significados que para generalizarlos. $Y$ a pesar de que los lectos de las diferentes generaciones continuarán divergiendo más y más, ambos extremos continuarán siendo inteligibles por un tiempo, siempre y cuando continúe la situación de contacto entre los idiomas en juego (criollo, inglés y español). Pero, ¿ha de continuar ese contacto?
Existe la posibilidad de que un nuevo proceso de creolización tenga lugar (criollo limonense-español), o que por el contrario se produzca un cambio total (en la terminología de Weinreich: "el cambio de un idioma por otro", 1974:68). La primera posibilidad parece remota porque las condiciones que se dan en Limón no parecen favorecer ese proceso (Edwards en su estudio de San Andrés, sugiere que se puede dar "en la ausencia de sistemas de autoridad que funcionan para mantener restricciones en las co-ocurrencias lingüísticas" 1970:249). Pero en Limón la situación es exactamente la opuesta. Por otra parte, predecir que el criollo morirá es una peligrosa aseveración.

Weinreich (1974:108) ha observado que "mu- 
chos idiomas obsoletos han recibido nuevas inyecciones de vida a través de una renovada lealtad al idioma, lo que hace la predicción de la muerte de un idioma un asunto peligroso." Además entran en esta especulación demasiados factores difíciles de controlar (aparte de los puramente lingüísticos, debería considerarse la dinámica del idioma en el tiempo, la función del idioma, el grado de cambio en las funciones, las actitudes subjetivas de los parlantes, el sexo, la afiliación religiosa, la política y la economía de la región, número de parlantes, sistema educativo, accesibilidad geográfica, etc., etc.).

En dos palabras, mientras más costarricenses blancos lleguen a Limón (actualmente existe una carretera por primera vez en la historia del país), y cuanto más deseen los miembros de la minoría integrarse a los sentimientos de nacionalismo, más posibilidades hay de que los parlantes reestructuren sus reglas para incorporar más y más elementos de prestigio en sus gramáticas. Podríamos preguntarnos ¿cuáles son las implicaciones para la teoría del lenguaje? ¿Podría ser que la relexificación sea total? ¿Se dará una nueva creolización? Y estos préstamos ¿qué significan en términos de la vida o muerte del criollo limonense? Estas son algunas de las preguntas que merecen ser estudiadas en el futuro.

\section{NOTAS}

(1) Las ideas expuestas en esta Introducción se basan especialmente en las observaciones de Michael D. Olien en "The Negro in Costa Rica: an Historical Perspective", MS, sin fecha.

(2) "Mamita Yunai" es el título de un libro escrito por Carlos Luis Fallas. "Mamita" representa la UFCo. y "Yunai" es la forma en que United se castellanizaba.

(3) El continuo va desde el extremo del criollo limonense (más cercano al criollo jamaicano de origen) hasta el otro, más próximo al inglés standard.

(4) Este trabajo se basa en un estudio anterior de mayor magnitud "Tense and Aspect in Limón Creole: a sociolinguistic view towar a creole continuuum." disertación de Ph. D. de la Universidad de Kansas, 1978.

(5) Limón es el nombre de la capital de la Provincia de Limón, ciudad donde se realizó este estudio.

(6) El nivel socioeconómico de la población no cuenta como variable en este caso ya que en realidad los estibadores, por ejemplo, perciben mejores salarios que otros trabajadores por el desgaste físico del trabajo pesado, sin embargo está claro que su salario no implica más educación.
(7) La forma que se adoptó para ilustrar los diferentes informantes es la siguiente: primero aparece el número de la cinta y su transcripción, luego el texto como fuera grabado.

(8) La división del continuo en basilecto, mesolecto y acrolecto es parte de la metodología que permite el estudio dinámico de los criollos (Stewart, Bickerton) se sobreentiende que la división es un instrumento enteramente artificial.

(9) Existieron en Limón desde fines del siglo XIX hasta 1953 escuelitas particulares que operaron con profesores importados de Jamaica para enseñarles a los niños "buen" inglés. Funcionaban bajo los auspicios de las iglesias o de la compañía bananera. Fueron cerradas por el gobierno para exigir la integración de la población negra a la cultura costarricense de habla española.

(10) Es probable que la penetración de un idioma en el otro pueda ser dilucidado alrededor de ciertos temas, tales como la educación, administración, el gobierno, los deportes, la cultura de la juventud, etc.

(11) A la larga, es posible que los vocablos españoles usados en el criollo limonense difieran en su alcance semántico de los usados en la meseta central.

\section{REFERENCIAS}

Bailey, Beryl Loftman. 1966. Jamaican Creole Syntax. Cambridge: Cambridge University Press.
Bickerton, Dereck. 1975. Dynamics of a Creole Syntax. Cambridge: Cambridge University Press. 
Bryce Laporte, Roy S. "Social Relations and Cultural Persistence (or change) among Jamaicans in a Rural Area of Costa Rica." Ph.D. Dissertation, University of Puerto Rico, 1962.

Cassidy, Frederic G. 1959. "English Language Studies in the Caribbean." American Speech, 34:163-171.

1961.Jamaica Talk: Three Hundred Years of the English Language in Jamaica. New York: St. Martin's Press.

and Le Page, R. B. 1967. Dictionary of Jamaican English. Cambridge: Cambridge University Press.

Diebold, A. Richard. 1964. "Incipient Bilingualism" in Dell Hymes (ed.), Language and Culture in Society: a Reader in Linguistics and Anthropology. New York: Harper and Row, pp. 495-508.

Edwards, Jay D. 1970. "Social Linguistics on San Andres and Providencia Island, Colombia." Ph.D. Dissertation, Tulane University.

Fallas, Carlos Luis. 1941. Mamita Yunai. San José: Editorial Soley y Valverde.

Haugen, Einar. 1956. The Bilingual Individual. "Bilingualism in the Americas: A Bibliography and Research Guide." Publication $\mathrm{N}^{\circ} 66$. American Dialect Sor ciety: University of Alabama Press, pp. 69-86.
Meléndez Chaverri, Carlos. 1966. "Los orígenes de los esclavos africanos en Costa Rica." Sevilla: XXXVI Congreso Internacional de Americanistas pp. 387-391.

Ministerio de Economía y Hacienda. 1953. Censo de Población de Costa Rica (22 mayo 1950). San José: Dirección General de Estadística y Censo.

Ministerio de Economía, Industria y Comercio. 1973. Censos Nacionales. Población. Tomos 1 y 2. San José: Dirección General de Estadística y Censos.

Olien, Michael D. n.d. "The Negro in Costa Rica: an Historical Perspective." MS.

Pride. J.B. 1971. The Social Meaning of Language. London: Oxford University Press.

Rayfield, J.R. 1970. The Languages of a Bilingual Community. The Hague: Mouton and Company.

Stewart, Watt. 1964. Keith and Costa Rica: A Biographical Study of Minor Cooper Keith. Albuquerque: The University of New Mexico Press.

Weinreich, Uriel. 1953. Languages in Contact. (8th printing, 1974). The Hague: Mouton and Company.

Zúñiga Tristán, Virginia. 1976. El Anglicismo en el Habla Costarricense. San José: Editorial Costa Rica, Editorial Universidad de Costa Rica. 